\section{Pay, Gender, and Pensions: High Time to Retire the Gender Pay Gap}

By Zara Nanu t's been 55 years since the passing of the Equal Pay Act in the United States and 48 years since a similar act was passed in the United Kingdom. Women who were entering the workforce at that time should now be retiring on equal footing as men. But nothing could not be further from the truth, and pay disparities are still very much a part of employment and retirement.

The World Economic Forum estimates the gender pay gap is at 60 percent globally, and it will take 217 years to close it. The World Economic Forum also estimates that, by 2030, most of us will be in self-driving cars, health care will be reshaped by technology and automation, and we will be on our way to the Red Planet. So, in 2030, while we are surrounded by this technological advancement, we will still be two centuries away from closing the gender pay gap. Surely we can leverage this technology to accelerate equality.

Increased access to data as well as various regulatory measures around the world mean that the gender pay gap is becoming an acknowledged fact. Most companies have one, and the reasons behind it are diverse. Moreover, the pay gap does not stop at the place of employment. It follows women into their pension age. This article will outline the current gender pay gap context and its impact on the pensions gap, and explore steps that can help us achieve parity faster.

The UK government recently introduced legislation that makes it mandatory for companies to analyze their gender pay gap and report six key figures to their stakeholders. When 10,736 companies reported their gaps in April 2018, the gender pay gap stopped being a myth and became a reality.

The only myths remaining are those that confuse the gender pay gap with equal pay. Equal pay means that men and women in the same employment performing equal or similar work must be remunerated equally. This is a legal requirement in most Western countries, and the United States is no exception. The Equal Pay Act of 1963 is a US labor law aimed at abolishing wage disparity based on sex. It was signed into law seven years before the UK Equal Pay Act.

The gender pay gap, on the other hand, is more about comparing average employees within a company, or an industry. It highlights discrepancies that are due to occupational segregation (women being largely present in lower-paid occupations and men in higher-paid occupations), lack of women in senior roles, what is known as the motherhood penalty, and a lack of flexibility and part-time opportunities in senior roles.

Although statistically we would use different methods to measure and understand these pay gaps, one thing is clear: these two notions are two sides of the same coin and need to be addressed together if we want to achieve earning parity in employment and retirement.

Why would we do that? Besides the ethical and moral implications of equality, the International Monetary Fund estimates that if women had equal access to the economy, Gross Domestic Product would increase by 5 percent in the United States, 9 percent in Japan, and 12 percent in the United Arab Emirates. ${ }^{1}$ Research by McKinsey estimates that closing the gender pay gap in the United Kingdom could add $£ 150$ billion to GDP by $2025 .^{2}$

Over the past 3 years at Gapsquare, our software has analyzed data that 


\section{"We are starting to see many interesting trends. One such trend that has predominated across industry, company size, and geography is that the gender pay gap starts to accelerate when women reach their 30 s and doubles with every decade in the workplace."}

cover more than 300,000 employees of different ages, occupations, experience levels, and job types. We are starting to see many interesting trends. One such trend that has predominated across industry, company size, and geography is that the gender pay gap starts to accelerate when women reach their 30 s and doubles with every decade in the workplace.

According to our aggregated data (as shown in the chart on the next page), women start with an average gender pay gap of 2 percent when they enter the workforce in their early 20 s. It increases to nearly 20 percent by the time they are in their 50s. A recent Equalities and Human Rights Commission study found the same when analyzing data from 1994, 2004, and 2014. ${ }^{3}$

The gender pay gap increases even more if women stay in the same workplace. After 3 years in a company, the gender pay gap - much like the age gap - begins to double every decade.

Research shows that the reasons behind this increase are similar to those outlined for age: women often take time off to care for young families, which in turn leads to slow (or no) career progression. These factors have an impact on women's earning capacity throughout their working lives, and women subsequently bring these inequalities with them into their pension age. ${ }^{4}$

Equal pay is one obvious answermen and women performing equal or similar work should be remunerated at the same level. But this will provide only half of the solution. The other half is addressing the gender pay gap.

Data indicate three key trending issues that can help narrow the gender pay gap faster: (a) occupational segregation, (b) shared parental leave, and (c) an overhaul of current remuneration and pay structures.

Occupational segregation is the clustering of certain workers with similar demographic characteristics - in this case, gender - in a specific occupation. The current workforce landscape still has jobs that are dominated by male workers and some roles that are dominated by female workers. This is important for this conversation because roles that are dominated by women pay less per hour than roles dominated by men. Nursing roles, for instance, pay less per hour than computer programming roles.
Because of this, women's earning capacity is diminished, and their retirement benefits are decreased. ${ }^{5}$ Occupational segregation is not something we can change overnight, but we can bring about a more conscious change when thinking about recruiting people into new roles.

Alongside our stereotypes and biases, the $\mathrm{CV}$ is another culprit in why occupational segregation continues to thrive. We live in a world where jobs are changing fast. Some of the jobs advertised on the job market today did not exist 20 years ago. Social media manager, data scientist, app developer, and cloud computing manager are jobs that have appeared in the past 10 years, and yet we still use a CV to recruit for such roles. Removing the CV from recruitment and focusing on a person's capacity to learn and adapt to the new role can help shift our mindset on occupational segregation.

Shared parental leave is instrumental in ensuring career breaks have the same impact on the careers of both men and women. Such career breaks are what cause the gender pay gap to increase after the age of $30 . \ln 2015$, the 


\section{Gender Pay Gap by Age and Tenure}

Mean Average Gender Pay Gap

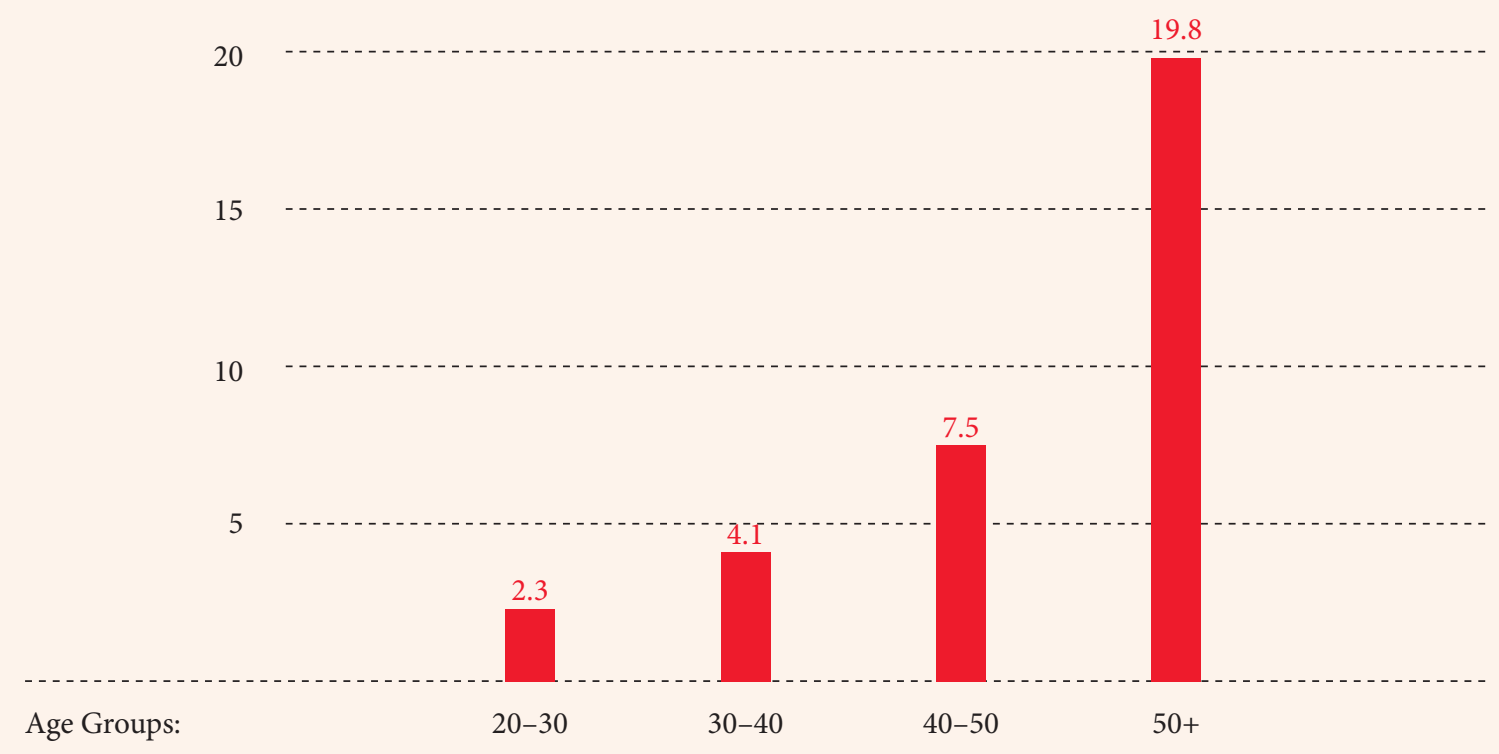

Mean Gender Pay Gap

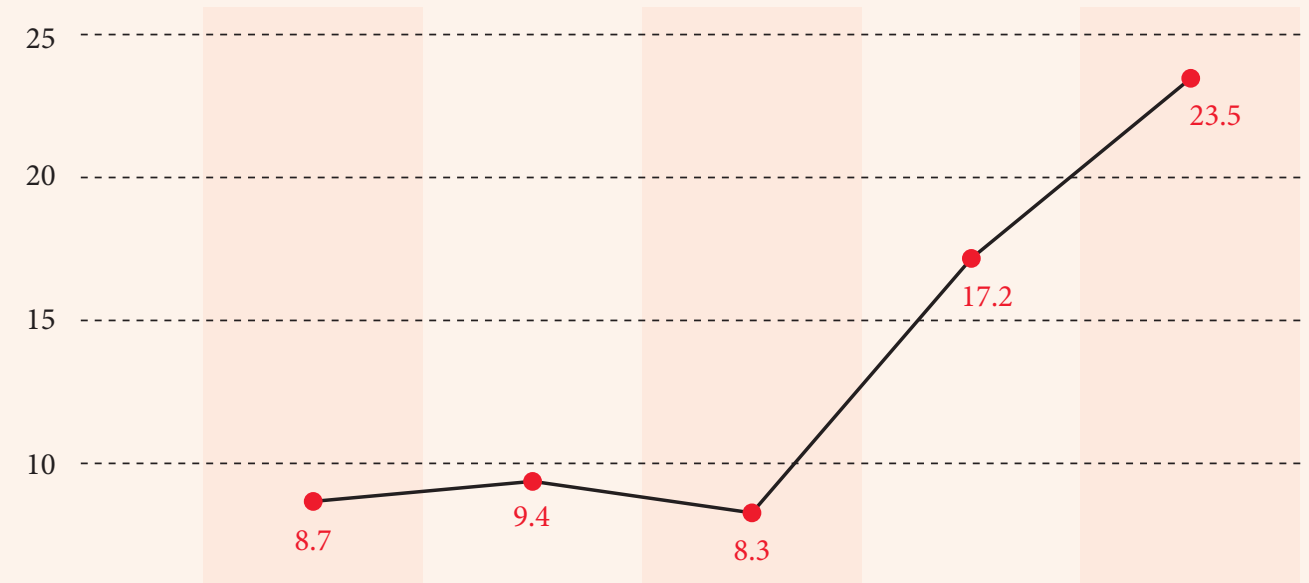

5 


\section{"If the gender pay gap is to close in the year 2235, per the World Economic Forum's estimate, it will take until at least 2270 to start receiving retirement benefits that would be free of pay disparities."}

United Kingdom saw the introduction of new rights allowing UK parents to share leave following the birth or adoption of their child. They allows parents to share up to 50 weeks of leave, 37 of which are paid. Scandinavian countries have such legislation providing even longer leave. As an example, Sweden offers 480 days per child, which parents can share as they wish. They are paid at a rate of about 80 percent of their salary.

The United Kingdom has only recently introduced shared parental leave, and the uptake of such leave among men still falls short of being equal. Encouraging more men to take these breaks can help accelerate equality for women.

Rethink pay structures and remuneration in line with the realities and requirements of the 21 st century. We live in a world of the digital workplace. Millennials and generations $X$ and $Y$ make up about 75 percent of the current workforce. They are motivated by fairness in pay, work-life balance, and a reflection of their values in their work. A 9-5 workplace is no longer suitable for these generations, and a remuneration structure that rewards a certain number of worked hours is dated. Instead, changes can be made to remunerate productivity and output, thus increasing uptake of part-time and flexible work. These changes will hit at the root causes of the gender pay gap.

It might seem that the changes outlined above require a momentous shift in working culture and mentality. Luckily, we live in an age in which technology is changing the way we travel, communicate, and learn. It can help us move on these recommendations with equally digital speed.

It's high time to retire the gender pay gap so that both men and women can enjoy their rights equally after they finish their professional careers.

1. M. Jovanović, "Girl Power," IMF Finance and Development 54, no. 1 (2017), https://www.imf.org/external/pubs/ft/ fandd/2017/03/pdf/picture.pdf.

2. McKinsey Global Institute, "The Power of Parity: Advancing Women's Equality in The United Kingdom" https://www.mckinsey.com/ /media/McKinsey/ Featured\%20Insights/Women\%20matter/The\%20 power\%20of\%20parity\%20Advancing\%20womens $\% 20$ equality\%20in\%20the\%20United\%20Kingdom/Powerof-parity-Advancing-womens-equality-in-the-UnitedKingdom-Executive-Summary.ashx

3. M. Brynin, The Gender Pay Gap (London: Equalities and Human Rights Commission, 2017, https://www. equalityhumanrights.com/sites/default/files/researchreport-109-the-gender-pay-gap.pdf.

4. Brynin, Gender Pay Gap.

5. Ariane Hegewisch and Emma Williams-Baron, "The Gender Wage Gap by Occupation 2017 and by Race and Ethnicity," Fact Sheet, Institute for Women's Policy Research, 2018.

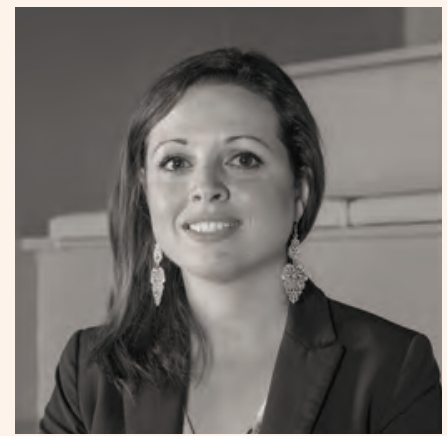

Zara Nanu

CO-FOUNDER AND CEO GAPSQUARE, UK 